\title{
The privilege and responsibility of caring for patients with rare genetic disorders
}

\author{
Debra E. Weese-Mayer ${ }^{1,2,3}$
}

Received: 15 January 2021 / Accepted: 17 January 2021 / Published online: 12 February 2021

○) Springer-Verlag GmbH Germany, part of Springer Nature 2021

Familial dysautonomia (FD), a rare hereditary sensory and autonomic neuropathy (HSAN-3) caused by a homozygous splicing mutation in intron 20 of the elongator complex protein 1 gene (ELPI or IKBKAP) [1], is characterized by presentation in infancy with impaired perception of pain and temperature and with autonomic nervous system dysregulation, including dopamine-related hypertensive crises with retching and vomiting.

In this issue of Clinical Autonomic Research, Ramprasad and colleagues raise visibility of the FD gastrointestinal phenotype, including enteric dysfunction, systematically assessed using a standardized survey instrument [2]. The motivations for this study were the anecdotal reports of gastrointestinal dysmotility, the fact that patients with FD have no or little pain despite overt gastrointestinal pathology, and the objective demonstration of carbidopa-responsive paroxysms of vomiting and retching with sympathetic surges. The authors aimed to establish the frequency and severity of gastrointestinal symptoms in patients with FD, which were identified as the most burdensome medical problem among respondents.

The article by Ramprasad and colleagues is a timely opportunity to address the importance, responsibility, and opportunities related to providing care to patients with rare genetic disorders, especially one that is followed longitudinally from childhood into adulthood, using FD to highlight successes and potential challenges. It is also timely that this

Debra E. Weese-Mayer

DWeese-Mayer@luriechildrens.org;

D-Weese-Mayer@ Northwestern.edu

1 Division of Pediatric Autonomic Medicine, Ann \& Robert H. Lurie Children's Hospital of Chicago, 225 East Chicago Avenue, Box 165, Chicago, IL 60611-2605, USA

2 Stanley Manne Children's Research Institute, Chicago, IL, USA

3 Department of Pediatrics, Northwestern University Feinberg School of Medicine, Chicago, IL, USA commentary is part of the special issue celebrating the 30th anniversary of Clinical Autonomic Research, useful to put into perspective discoveries about rare genetic disorders over the past decades.

Though described nearly 75 years ago, and despite fewer than 400 individuals currently living with FD worldwide, FD serves as an inspiring model for other rare conditions. Dr. Felicia Axelrod established the Dysautonomia Center at the New York University (NYU) School of Medicine more than four decades ago, recognizing the privilege and responsibility of providing care to fragile, typically pediatric patients with this rare condition. She partnered with genetic experts, key among them Dr. Susan Slaugenhaupt, in a quest to identify the genetic basis of FD, which she found. Dr. Axelrod recognized that successfully building and sustaining a cohort of patients with a rare disease is invaluable for affected patients as well as for the basic science community who shares the responsibility of studying the basic underpinnings of that rare disease with aim to diminish symptoms. The success of the NYU FD Patient Registry (established in 1980; including demographic and medical information for all genetically confirmed patients with FD who received care at the NYU Langone Medical Center) is the result of foresight and shared credit due to the dedication of Dr. Axelrod, the parents who developed and support the Familial Dysautonomia Foundation, and countless other parents, grandparents and family members whose lives are forever touched by a child—or adult—with FD and who continue to provide funding for the FD clinical and research program at NYU, now led by Dr. Horacio Kaufmann. Dr. Kaufman has expertly shepherded FD into a new era, building on the success of the prior era, and methodically, innovatively, and successfully advancing the science of FD. The result is exciting and the development of a disease-modifying treatment, either with RNA-based technology or other types of gene therapies, for FD should be close. This is something that no one might have dared whisper 10 or 15 years ago. While preimplantation genetics has resulted in fewer newborns 
diagnosed with FD, there are still two or three babies diagnosed with FD each year, and also adult patients who require care and who continue to inform the scientific community as to the natural history of FD, consequently motivating clinician-researchers to identify ways to decrease disease burden and to inform regarding other potential HSANs.

In careful review of the contribution of Ramprasad and colleagues in this issue of Clinical Autonomic Research, it is noteworthy that the authors struggled with the following issues: (1) finding a proper age-matched control group for the patients with FD; (2) identifying a gastrointestinal symptom instrument appropriate for patients from childhood to adulthood; (3) determining cognitive function in patients with FD; (4) assessing gastrointestinal symptoms in patients with cognitive impairment; and (5) interpreting gastrointestinal symptoms in a cohort of FD patients, where the standard of care for decades was a fundoplication.

Because the validated National Institutes of Health Patient-Reported Outcomes Measurement Information System (PROMIS) survey questionnaire [3] is an adult database, the authors had to exclude the pediatric FD patients from the final analysis. The MoCA scale [4] (MoCA), designed for adults with dementia, was used to screen for cognitive impairment in the FD cohort, so it had limited application to the younger patients. Though the above-described issues are specific to FD, they have relevance to any study focused on a specific organ system in patients with rare diseases. Also broadly applicable to FD and other rare genetic disorders is the potential for introduction of the NIH Toolbox [5] that might serve as an ongoing measure to objectively identify cognitive impairment in patients in the 3-84 year age range, with built-in controls.

Again, with focus on FD but with application to other rare genetic disorders, there are two missed opportunities: (1) the development of an ongoing healthy-matched control cohort to follow longitudinally and (2) the prospective administration of the same instruments longitudinally in the patients and controls to identify the disease trajectory. In other words, healthy age-matched controls should be part of disease-specific natural history studies. Addressing these two opportunities would incur substantial time and expense but would together allow for objective questionnaire and physiologic data to be followed with advancing age, serving as a basis for trajectory analysis. Only through this strategy will the investigators be able to confidently assess the phenotype of cases and controls longitudinally, and to use the identified differences to allow for anticipatory management with the aim to decrease the disease burden.
While it is remarkable that the FD cohort included 202 living patients, the $38 \%$ response rate was subpar but fortuitously representative of the overall FD cohort followed at NYU. The take-home message of the article by Ramprasad and colleagues is that nearly all patients with FD have gastrointestinal symptoms and that overall, these gastrointestinal symptoms are more prevalent, but the severity reduced, compared to the PROMIS adult controls.

In addition, the take-home message of this editorial comment is twofold: (1) a clearer understanding of the gastrointestinal phenotype in FD allows for further investigations on its pathophysiology to decrease the symptomatic burden in FD and (2) there is an opportunity to apply the lessons learned in FD to advance the science and healthcare for patients with other rare genetic disorders, with the potential of a universal gain.

Funding None.

\section{Compliance with ethical standards}

Conflict of interest The authors declare that they have no conflict of interest.

\section{References}

1. Slaugenhaupt SA, Blumenfeld A, Gill SP, Leyne M, Mull J, Cuajungco MP, Liebert CB, Chadwick B, Idelson M, Reznik L, Robbins C, Makalowska I, Brownstein M, Krappmann D, Scheidereit C, Maayan C, Axelrod FB, Gusella JF (2001) Tissue-specific expression of a splicing mutation in the IKBKAP gene causes familial dysautonomia. Am J Hum Genet 68:598-605

2. Ramprasad C, Norcliffe-Kaufmann L, Palma J-A, Levy J, Zhang Y, Spalink CL, Khan A, Smukalla S, Kaufmann H, Chen LA (2021) Frequency and burden of gastrointestinal symptoms in familial dysautonomia. Clin Auton Res. https://doi.org/10.1007/ s10286-020-00735-9

3. Almario CV, Ballal ML, Chey WD, Nordstrom C, Khanna D, Spiegel BMR (2018) Burden of gastrointestinal symptoms in the United States: results of a nationally representative survey of over 71,000 Americans. Am J Gastroenterol 113:1701-1710

4. Nasreddine ZS, Phillips NA, Bedirian V, Charbonneau S, Whitehead V, Collin I, Cummings JL, Chertkow H (2005) The Montreal Cognitive Assessment, MoCA: a brief screening tool for mild cognitive impairment. J Am Geriatr Soc 53:695-699

5. Gershon RC, Wagster MV, Hendrie HC, Fox NA, Cook KF (2013) Nowinski CJ (2013) NIH toolbox for assessment of neurological and behavioral function. Neurology 80(11 Suppl 3):S2-6 